\title{
Lung-targeting drug delivery system of baicalin- loaded nanoliposomes: development, biodistribution in rabbits, and pharmacodynamics in nude mice bearing orthotopic human lung cancer
}

\author{
This article was published in the following Dove Press journal: \\ International Journal of Nanomedicine \\ 29 December 2016 \\ Number of times this article has been viewed
}

\author{
Yumeng Wei' \\ Jing Liang' \\ Xiaoli Zheng ${ }^{2}$ \\ Chao $\mathrm{Pi}^{1}$ \\ Hao Liu' \\ Hongru Yang ${ }^{3}$ \\ Yonggen Zou ${ }^{4}$ \\ Yun Ye ${ }^{1,5}$ \\ Ling Zhao' \\ 'Department of Pharmaceutics, \\ School of Pharmacy, Southwest \\ Medical University, ${ }^{2}$ Department \\ of Biochemistry, The Institute of \\ Basic Medical Sciences, Southwest \\ Medical University, Jiangyang District, \\ ${ }^{3}$ Department of Oncology, The \\ Affiliated Hospital of Southwest \\ Medical University, ${ }^{4}$ Department of \\ Orthopedics, The Affiliated Hospital \\ of Traditional Chinese Medicine \\ of Southwest Medical University, \\ Longma Tan District, ${ }^{5}$ Department of \\ Pharmacy, The Affiliated Hospital of \\ Southwest Medical University, Luzhou \\ City, Sichuan Province, People's \\ Republic of China
}

Correspondence: Ling Zhao Department of Pharmaceutics, School of Pharmacy, Southwest Medical University, No 3-319, Zhongshan Road, Jiangyang District, Luzhou City, Sichuan Province 646000, People's Republic of China Tel/fax +86830 316 229l Email zhaoling-998@163.com

\begin{abstract}
The present study aims to develop a kind of novel nanoliposomes for the lung-targeting delivery system of baicalin as a Chinese medicine monomer. Baicalin-loaded nanoliposomes were prepared by the effervescent dispersion and lyophilized techniques. Baicalin-loaded nanoliposomes had an average particle size of $131.7 \pm 11.7 \mathrm{~nm}$ with $0.19 \pm 0.02$ polydispersity index, $82.8 \% \pm 1.24 \%$ entrapment efficiency and $90.47 \% \pm 0.93 \%$ of yield and sustaining drug release effect over $24 \mathrm{~h}$ and were stable for 12 months at least. In vitro no hemolytic activity was observed for the experimental drug concentration. After intravenous administration of baicalin-loaded nanoliposomes to rabbits, drug concentration in the lungs was the highest among the tested organs at all time points and was significantly higher than that of its solution. For the targeting parameters, the relative intake rate and the ratio of peak concentration of lung were 4.837 and 2.789, respectively. Compared with plasma, liver, spleen, and kidney, the ratios of targeting efficacy $\left(\mathrm{T}_{\mathrm{e}}\right)_{\text {liposomes }}$ to $\left(\mathrm{T}_{\mathrm{e}}\right)_{\text {injection }}$ of lung were increased by a factor of $14.131,1.893$, 3.357, and 3.470, respectively. Furthermore, the results showed that the baicalin-loaded nanoliposomes did not induce lung injury. Importantly, baicalin-loaded nanoliposomes showed better antitumor therapeutic efficacy in the nude mice bearing orthotopic human lung cancer with the median survival time of blank liposomes ( $11.40 \pm 0.16$ days), baicalin solution (17.30 \pm 0.47 days), and baicalin-loaded nanoliposomes ( $25.90 \pm 0.53$ days). Therefore, the liposome is a promising drug carrier with an excellent lung-targeting property and therapeutic effect for the treatment of lung disease, such as lung cancer.
\end{abstract}

Keywords: liposomes, biodistribution, lung-targeting drug delivery, cancer therapy, baicalin

\section{Introduction}

According to the World Health Organization, lung cancer is the leading cause of cancer-related death. ${ }^{1,2}$ It was estimated that 1.37 million deaths were caused by lung cancer worldwide. ${ }^{3}$ Although there are some improvements in lung cancer therapy, the 5-year survival rate is as low as $15.9 \%$ for all stages combined. ${ }^{4}$ At present, chemotherapy, surgery, and radiotherapy are the main methods for the treatment of lung cancer. Unfortunately, $75 \%$ of patients with lung cancer diagnosed at the first time are found to have advanced disease that is incurable and lose the chance of accepting surgical treatment. ${ }^{4}$ Therefore, it was suggested that chemotherapy should be the most important measure in the treatment for patients with advanced stage and inoperable lung cancer. However, because most of drugs in conventional dosage forms are distributed 
throughout the body, the treatment of lung cancer is ineffective and results in serious side effects. ${ }^{3}$ Thus, lung-targeted drug delivery systems (LTDDS) have been recognized as an ideal strategy for the treatment of lung cancer. ${ }^{5}$

Baicalin is 7-D-glucuronic acid 5,6-dihydroxyflavone derived from the dried root of Scutellaria baicalensis Georgi, known as radix scutellariae, a basic and key medicinal composition unit in the traditional Chinese medicine. ${ }^{6,7}$ Extensive studies have shown that baicalin has strong antitumor effects. ${ }^{8-10}$ For the treatment of lung cancer, in vitro experiments, baicalin could suppress cell proliferation of human lung cancer A549 and mouse Lewis lung cancer in a dose- and time-dependent behavior. In in vivo study, baicalin could reduce tumor growth and prolong survival period in C57BL/6 mice bearing Lewis lung carcinoma tumor and nude mice bearing A549 carcinoma. ${ }^{11}$ In order to improve treatment efficacy of baicalin in lung cancer, it is necessary to develop LTDDS for baicalin.

The LTDDS mainly include liposomes, microspheres and nanoparticles via intravenous route. ${ }^{5,12-17}$ From the biomedical point of view, the liposomes composed of phospholipids and cholesterol are nontoxic, biocompatible, and biodegradable drug carriers. Liposomes as LTDDS have been extensively studied and have attracted increasing attentions in the past few decades. ${ }^{12,13}$ To date, no studies have been reported with respect to the LTDDS for baicalin. In this study, lungtargeting baicalin-loaded nanoliposomes were developed for the first time. For in vitro evaluation, baicalin-loaded nanoliposomes were evaluated in terms of mean size, polydispersity index (PDI), encapsulation efficiency, yield, in vitro release, stability, and hemolytic study. For in vivo evaluation, tissue distribution and lung-targeting characterization in rabbits were investigated after intravenous administration of baicalin-loaded nanoliposomes and its solutions as the control. Besides, we also evaluated the potential lung injury induced by baicalin-loaded nanoliposomes in rats and investigated pharmacodynamics in nude mice bearing orthotopic human lung cancer for the first time.

\section{Materials and methods \\ Materials}

The reference substance of baicalin used in the analysis was obtained from the National Institutes for Food and Drug Control of China (Beijing, China). The general baicalin was purchased from Xinxiang Bokai Bio-Technology Co., Ltd. (Henan, China). Rutin used as internal standard was obtained from Chengdu Mansite Pharmaceutical Co., Ltd. (Sichuan, China). Phosopholipon 90H (HSPC) was obtained from
Shanghai Toshisun Bio-Technology Co., Ltd. (Shanghai, China). Matrigel was purchased from Shanghai Shenxiang Co., Ltd. (Shanghai, China). Citric acid (injection grade), carbonic acid monosodium salt $\left(\mathrm{NaHCO}_{3}\right)$, Tween-80 (injection grade), dehydrated alcohol, and ammonium acetate were obtained from Luzhou Juhe Chemical Co., Ltd., (Luzhou, China). Acetonitrile and methanol (high-performance liquid chromatography [HPLC] grade) were obtained from Chengdu Jinghong Co., Ltd., (Chengdu, China). In all, 0.9\% of sodium chloride injection was supplied from the Affiliated Hospital of Southwest Medical University (Luzhou, China). Ultrapure water used in this study was produced by water purification system in the laboratory.

\section{Cells and animals}

Human lung cancer A549 cells were purchased from Cell Bank, Shanghai Institutes for Biological Sciences, Chinese Academy of Sciences (Shanghai, China). The cells were cultured in the Roswell Park Memorial Institute 1640 medium containing $10 \%$ fetal bovine serum, streptomycin $(100 \mu \mathrm{g} / \mathrm{mL})$, and penicillin $(100 \mathrm{U} / \mathrm{mL})$ in a humidified $5 \%$ $\mathrm{CO}_{2}$ atmosphere at $37^{\circ} \mathrm{C}$.

New Zealand rabbits weighing 1.5-2.0 kg and Sprague Dawley rats weighing 150-200 g were provided by the Laboratory Animal Center of Southwest Medical University (Luzhou, China). The Balb/c nude mice in the weight range of 17-20 g were obtained from Jianyang Dashuo Biology Technology Co., Ltd. (Sichuan, China). The animals were housed in a temperature- and moisture-controlled $\left(20^{\circ} \mathrm{C} \pm 2^{\circ} \mathrm{C}\right.$ and $55 \% \pm 10 \%$, respectively) room with a 12 -h light-dark cycle and allowed free access to food and water. They were fasted for $12 \mathrm{~h}$ before experiment. All procedures involving animals complied with the requirements of the National Act on the Use of Experimental Animals (China). This study was approved by the Southwest Medical University Animal Ethical Experimentation Committee (No 2013002).

\section{Preparation of baicalin-loaded nanoliposomes}

Baicalin-loaded nanoliposomes were prepared by the effervescent dispersion technique. Briefly, baicalin, HSPC, Tween-80, and citric acid (mass ratio $=2: 2: 1: 1$, respectively) were dissolved in ethanol. The solution was added dropwise to $\mathrm{NaHCO}_{3}$ water solution $(0.5 \%, \mathrm{w} / \mathrm{v})$ containing mannitol $(5.0 \%, \mathrm{w} / \mathrm{v})$ at $15^{\circ} \mathrm{C}-20^{\circ} \mathrm{C}$ under continuous stirring. The mixture solution was continuously stirred until it became cheese like. Finally, the cheese-like products were lyophilized and stored at $2^{\circ} \mathrm{C}-8^{\circ} \mathrm{C}$ for further investigation. 


\section{Particle size and PDI}

The mean diameter and size distribution (PDI) of baicalinloaded nanoliposomes were measured by NanoBrook 90Plus Zeta (Brookhaven Instruments, Holtsville, NY, USA) at $25^{\circ} \mathrm{C}$. Before measurement, weighed freeze-dried powder was briefly dispersed in the ultrapure water and then was diluted with ultrapure water to achieve a suitable scattering intensity. Each sample was measured in triplicate.

\section{Entrapment efficiency}

The entrapment efficiency (EE) was determined by the equilibrium dialysis method with dialysis membranes (cutoff 8,000-10,000; Luzhou Kelong Reagent Co. Ltd., China). The samples of baicalin-loaded nanoliposomes were dispersed with ultrapure water and then dialyzed against phosphatebuffered saline (PBS; pH 7.4) for $4 \mathrm{~h}$. After dialysis, free drug was determined by the HPLC. An aliquot of $0.2 \mathrm{~mL}$ of baicalin-loaded nanoliposome suspension was vortex mixed with $0.8 \mathrm{~mL}$ of methanol for $5 \mathrm{~min}$. Afterward, the supernatant following centrifuging was measured by HPLC to obtain the total drug content in baicalin-loaded nanoliposome sample.

Briefly, HPLC was performed by using chromatographic system (pump: LPG-3400SD, UV-vis detector: VWD-3100, auto injector: WPS-3000 and column oven: TCC-3000, C18 column: Inertsil ODS-SP $[4.6 \times 250 \mathrm{~mm}, 5 \mu \mathrm{m}$ particle size, made in Japan] and guard column: Phenomenex C18 [4.0 $\times 3.0 \mathrm{~mm}, 5 \mu \mathrm{m}$ particle size, made in the USA]). The methanol, acetonitrile, and $0.4 \%(\mathrm{v} / \mathrm{v})$ aqueous phosphoric acid (7.5:7.5:85, v/v, respectively) were chosen as a mobile phase at the detection wavelength of $278 \mathrm{~nm}$. The flow rate was $1.0 \mathrm{~mL} / \mathrm{min}$ at $35^{\circ} \mathrm{C}$, and the injection volume was $20 \mu \mathrm{L}$.

The EE\% was calculated by the following equation:

$$
\mathrm{EE} \%=\frac{\mathrm{W}_{\text {total }}-\mathrm{W}_{\text {free }}}{\mathrm{W}_{\text {total }}} \times 100 \%
$$

$\mathrm{W}_{\text {free }}$ was the amount of free baicalin in liposomal samples, and $\mathrm{W}_{\text {total }}$ was the total drug amount in baicalin nanoliposomal formulation.

\section{In vitro release}

Baicalin released from its nanoliposomal formulation was evaluated by dialysis. The freeze-dried powder of baicalinloaded nanoliposomes was redissolved with ultrapure water to obtain an appropriate drug concentration (baicalin, $7.5 \mathrm{mg} / \mathrm{mL}$ ) and sealed in dialysis bags (cutoff 8,000-10,000;
Luzhou Kelong Reagent Co. Ltd.). Then the samples were dialyzed against $100 \mathrm{~mL}$ of $\mathrm{PBS}(\mathrm{pH} 7.4)$ as a release medium at a stirring rate of $100 \mathrm{rpm}$ at $37^{\circ} \mathrm{C}$. At predetermined time interval, $0.2 \mathrm{~mL}$ of release medium was collected and complemented with fresh PBS buffer at the same temperature. The supernatant obtained by centrifuging the sample solution was determined by the HPLC system as described earlier.

\section{Stability studies}

Dried baicalin-loaded nanoliposome samples were sealed at $4^{\circ} \mathrm{C}$ for 12 months. The samples were analyzed for the changes in particle size and EE at fixed time intervals, respectively.

\section{Hemolytic study}

Whole blood was obtained from the rabbit and stirred to remove fibrinogen by a glass stick. Afterward, red blood cells (RBCs) obtained by centrifuging at $1,000 \mathrm{rpm}$ for 10 min were washed by normal saline. The same course was repeated to obtain clear and colorless supernatant. The RBCs were resuspended in normal saline to reach $5 \%(\mathrm{w} / \mathrm{v})$ cell suspension and used further for hemolytic evaluation. The different volumes of baicalin nanoliposome suspension were added to $2.5 \mathrm{~mL}$ of the RBCs. The final volume was adjusted to $5 \mathrm{~mL}$ with normal saline. In addition, $2.5 \mathrm{~mL}$ of the RBCs treated with $2.5 \mathrm{~mL}$ of normal saline was considered as negative control, while water was considered as positive control. The mixtures were incubated at $37^{\circ} \mathrm{C}$ for $0.5,1.5$, and $3 \mathrm{~h}$ and then centrifuged at 1,000 rpm. The absorbance values of the supernatants were measured at $570 \mathrm{~nm}$ by a spectrophotometer (Thermo Fisher Scientific, Waltham, MA, USA). The percentage of hemolysis was calculated by the following equation:

$$
\text { Percentage of hemolysis }=\frac{A b s-A b_{0}}{A b_{100}-A b_{0}} \times 100
$$

Abs and $\mathrm{Ab}_{0}$ are absorbance values of the baicalin-loaded nanoliposome sample and negative control group, and $\mathrm{Ab}_{100}$ is the value of the positive control group.

\section{Tissue distribution and lung-targeting evaluation}

The rabbits were randomly divided into ten groups with five each. Baicalin-loaded nanoliposomes or its solutions as the control were administered intravenously from group 1 to group 5 or from group 6 to group 10 through their ear marginal vein with a single dose of $10 \mathrm{mg} / \mathrm{kg}$ body weight of baicalin. At $0.25,0.5,1,1.5,2,4,6,8,12$, and $24 \mathrm{~h}$ after 
the intravenous administration, the whole blood samples were collected and immediately centrifuged at 5,000 rpm for $10 \mathrm{~min}$ to obtain plasma samples.

Afterward, the rabbits were sacrificed and heart, liver, spleen, lung, kidney, stomach, and brain were immediately removed at each time point and washed with $0.9 \%$ saline solution. The tissue samples were treated with filter paper to remove the redundant liquid, weighed, and homogenized. All plasma and tissue samples were frozen at $-20^{\circ} \mathrm{C}$ until analyzed by HPLC.

In all, $50 \mu \mathrm{L}$ of rutin internal standard solution containing $80.0 \mu \mathrm{g} / \mathrm{mL}$ of methanol and $250 \mu \mathrm{L}$ of ammonium acetate buffer ( $\mathrm{pH} 3.5 ; 1 \mathrm{M})$ were added to $0.5 \mathrm{~mL}$ of plasma or tissue homogenate samples in a centrifuge tube with $10 \mathrm{~mL}$ capacity and vortexed for $3 \mathrm{~min}$. Then, $3 \mathrm{~mL}$ of acetonitrile was added to the solution by vortex mixing for $5 \mathrm{~min}$. The clear supernatant obtained by centrifuging at 10,000 rpm for 10 min was collected and evaporated to dryness under nitrogen gas stream at $40^{\circ} \mathrm{C}$. The residue was then resuspended in $200 \mu \mathrm{L}$ of mobile phase and centrifuged at 10,000 rpm for $10 \mathrm{~min}$. Then, $20 \mu \mathrm{L}$ of the clear supernatant was injected into the HPLC system.

For the determination of baicalin in plasma and tissues, HPLC was performed using UltiMate 3000 series chromatographic system (Dionex, CA, USA) with separation on a reverse-phase C18 column Inertsil ODS-SP $(4.6 \times 250 \mathrm{~mm}$, $5 \mu \mathrm{m})$ protected by a Phenomenex C18 guard column $(4.0 \times 3.0 \mathrm{~mm}, 5 \mu \mathrm{m})$. The mobile phase consisted of the mixture of methanol and acetonitrile $(1: 1, \mathrm{v} / \mathrm{v})(\mathrm{A})$ and $0.4 \%$ $(\mathrm{v} / \mathrm{v})$ aqueous phosphoric acid (B) using a gradient elution of $85 \% \mathrm{~B}$ at $0-1 \mathrm{~min}, 85 \%-30 \% \mathrm{~B}$ at $1-14 \mathrm{~min}$ and $30 \%-85 \%$ $\mathrm{B}$ at $14-15 \mathrm{~min}$. The flow rate was $1.0 \mathrm{~mL} / \mathrm{min}$, and the column temperature was maintained at $35^{\circ} \mathrm{C}$. In all, $20 \mu \mathrm{L}$ of sample solution was injected into HPLC and assayed at the wavelength of $278 \mathrm{~nm}$.

On the basis of pharmacokinetic parameters obtained in this study, three targeting parameters, namely, the intake rate $\left(\mathrm{R}_{\mathrm{e}}\right)$, targeting efficacy $\left(\mathrm{T}_{\mathrm{e}}\right)$, and peak concentration ratio $\left(\mathrm{C}_{\mathrm{e}}\right)$ were collected to evaluate the lung-targeting effect of baicalin-loaded nanoliposomes.

\section{Evaluation of lung injury}

Rats were randomly allocated into two groups with equal gender ratio. Both experimental groups received baicalinloaded nanoliposomes via tail vein injection with a single dose of $100 \mathrm{mg} / \mathrm{kg}$ body weight of baicalin, while the control group received the same volume of normal saline. For each group, the rats were sacrificed immediately at the first, fourth, and seventh days after injection. Then lung tissues were removed and washed with cold saline. The lung tissues were fixed in $10 \%$ neutral formalin, embedded in paraffin, sliced at a thickness of $5 \mu \mathrm{m}$ and stained with hematoxylin and eosin (HE). The histopathological studies were carried out to identify lung injury induced by LTDDS for baicalinloaded nanoliposomes.

\section{Pharmacodynamics}

The Balb/c nude mice were anesthetized using intraperitoneal injection of pentobarbital sodium solution at a dose of $50 \mathrm{mg} / \mathrm{kg}$ body weight. Afterward, $1.0 \times 10^{7}$ human lung cancer A549 cells in the logarithmic growth phase suspended in $100 \mu \mathrm{L}$ of PBS and Matrigel $(1: 1, \mathrm{v} / \mathrm{v})$ were injected into right lungs of mice guided by multislice computed tomography (CT). At 7 days after tumor transplantation, the mice were scanned by high-resolution CT. Among the mice, three of them were randomly collected and sacrificed. Then lung was immediately removed and fixed in $10 \%$ (v/v) neutral-buffered formalin to process for HE staining. The results showed that the xenotransplantation model of human lung cancer A549 cells could be successfully established by this method.

On day 7 , the nude mice bearing orthotopic human lung cancer were randomly divided into three groups. Each group $(\mathrm{n}=10)$ was injected intravenously with blank nanoliposomes (0 mg baicalin/ $\mathrm{kg}$ body weight), baicalin solution (100 mg baicalin/kg body weight), and baicalin nanoliposomes (100 mg baicalin/kg body weight) at the same volume. The treatment of each group was started at 7 days after tumor transplantation, and they continued to die naturally on every other day. During the experimental process, when the mice naturally scarified, lung tumor was excised immediately and weighed accurately.

\section{Data analysis}

On the basis of the changes in drug concentration of plasma and tissues as a function time, pharmacology software Drug and Statistics Software 2.0 (Mathematical Pharmacology Professional Committee of China, Shanghai, China) was used to calculate pharmacokinetic parameters. Survival comparison between groups was conducted with Kaplan-Meier survival curves. All results were expressed as mean \pm standard deviation (SD), and statistically significant difference between baicalin-loaded nanoliposomes and its injectable solution as control was evaluated using two-tailed $t$-test. The level of significant difference was set at $P<0.05$. 


\section{Results and discussion Characteristics of baicalin-loaded nanoliposomes}

After freeze-drying of baicalin nanoliposomal formulation, the yellow and loose powder or granules were obtained. On the basis of the optimized formulation and technology, three batch samples of baicalin-loaded nanoliposomes were prepared and characterized in terms of particle size, PDI, zeta potential, EE, and yield (Table 1). Particle size and PDI of baicalin-loaded nanoliposomes measured by NanoBrook 90Plus Zeta were $131.7 \pm 11.7 \mathrm{~nm}$ and $0.19 \pm 0.02$, respectively. The EE of baicalin-loaded nanoliposomes was $82.8 \% \pm 1.24 \%$, which was higher than that $(34.62 \%-60.11 \%)$ of other results reported in the literature. Hong et $\mathrm{al}^{18}$ and Chen et al ${ }^{19}$ developed baicalin-loaded nanoliposomes and flexible nanoliposomes with EE of $34.62 \%$ and $60.11 \%$, respectively. It was well known that there was a significant difference in EE of liposomes prepared by different formulations and preparation technologies.

\section{In vitro release behavior}

In the present study, dialysis bag method was chosen to investigate the in vitro release behavior of baicalin-loaded nanoliposomes. The results of in vitro release of baicalin from liposomal formulation and its solution (baicalin was dissolved in $0.1 \% \mathrm{NaOH}$ and then $\mathrm{HCl}$ was used to adjust the $\mathrm{pH}$ value to be 7.3) as control are presented in Figure 1. As shown in Figure 1, baicalin liposomal formulation exhibited a slower release behavior in comparison with its solution. Baicalin released from the liposome carrier was 37.8\% $\pm 1.9 \%$, $78.5 \% \pm 2.9 \%$, and $89.6 \% \pm 2.1 \%$ at 2,12 , and $24 \mathrm{~h}$ of dialysis, respectively, whereas, baicalin released from its solution was $90.0 \% \pm 3.6 \%$ at $4 \mathrm{~h}$. A similar trend in the vitro release behavior for baicalin-loaded nanoliposomes prepared in this study was observed compared to results reported previously. ${ }^{20,21}$ No significant difference in the rate of drug release in the case of three batch samples of baicalin-loaded nanoliposomes was observed $(P>0.05)$. It further suggested that the optimal formulation possessed of a good reproduction.

Table I The characterization of three batch samples of baicalinloaded nanoliposomes

\begin{tabular}{lllll}
\hline Batch & Mean size $(\mathrm{nm})$ & PDI & EE (\%) & Yield (\%) \\
\hline $\mathrm{I}$ & $129.7 \pm 13.5$ & $0.20 \pm 0.02$ & $82.6 \pm 1.09$ & 91.5 \\
2 & $137.9 \pm 10.5$ & $0.18 \pm 0.02$ & $83.8 \pm 1.35$ & 90.2 \\
3 & $129.9 \pm 8.8$ & $0.20 \pm 0.02$ & $81.9 \pm 1.29$ & 89.7 \\
\hline
\end{tabular}

Note: Data presented as mean \pm standard deviation.

Abbreviations: PDI, polydispersity index; EE, entrapment efficiency.

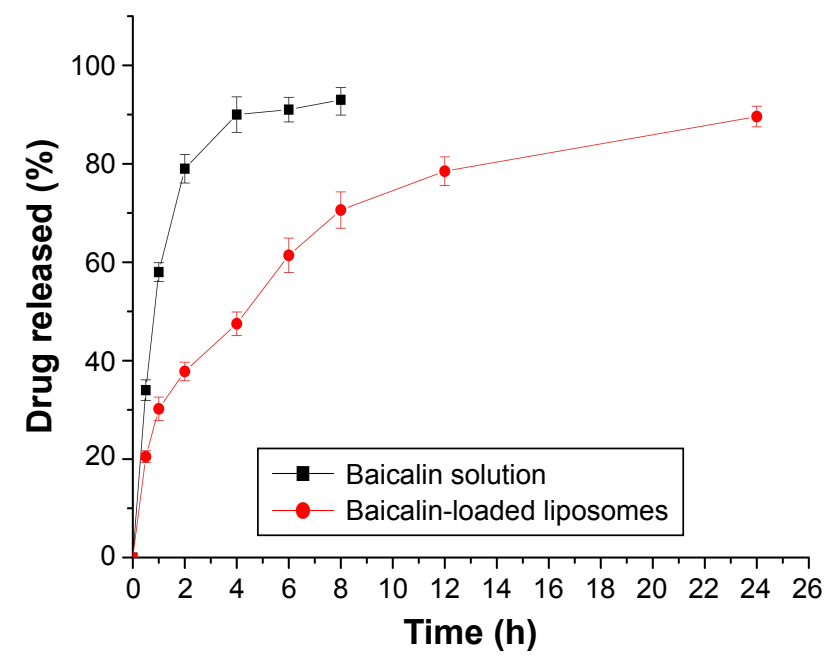

Figure I Cumulative percent drug release from baicalin-loaded nanoliposomes and its solution $(n=3)$.

\section{Stability studies}

To determine whether baicalin liposomes as freeze-dried powder is more stable, baicalin-loaded nanoliposomes were stored at $4^{\circ} \mathrm{C}$ over a period of 12 months and sampled periodically to determine the particle size and $\mathrm{EE} \%$ of the drug. The stability test of baicalin-loaded nanoliposomes is summarized in Table 2. Although a slight change was observed, namely, the mean particle size increased and EE $(\%)$ decreased as the storage time prolonged, the results demonstrated that the mean particle size and EE (\%) had no significant change during 12 months $(P>0.05)$.

\section{Hemolytic study}

It is well known that hemolytic activity is an important factor to investigate the quality of new drug dosage form for intravenous administration. As shown in Figure 2, baicalin liposomal formulation developed in the present study was found to possess negligible hemolysis of erythrocytes. Therefore, the encapsulation of baicalin in liposome carriers could reduce its hemolytic toxicity to a very low level. For example, maximum drug concentration at a dose of $4.5 \mathrm{mg} / \mathrm{mL}$ resulted in $<5 \%$ hemolysis rate.

\section{Tissue distribution and lung-targeting evaluation}

In order to prove lung-targeting characterization, the in vivo tissue distribution of baicalin was performed after intravenous administration of baicalin-loaded nanoliposomes and its solution as the control with a single dose of $10 \mathrm{mg} / \mathrm{kg}$ to rabbits; the results are summarized in Figures 3 and 4. As shown in 
Table 2 The stability of baicalin-loaded nanoliposomes (mean \pm SD, $n=3$ )

\begin{tabular}{llllllll}
\hline Time (months) & $\mathbf{0}$ & $\mathbf{1}$ & $\mathbf{2}$ & $\mathbf{3}$ & $\mathbf{6}$ & $\mathbf{9}$ & $\mathbf{1 2}$ \\
\hline $\mathrm{EE}(\%)$ & $82.8 \pm 1.24$ & $82.6 \pm 1.28$ & $82.5 \pm 1.26$ & $82.3 \pm 1.52$ & $81.9 \pm 1.79$ & $81.5 \pm 2.02$ & $81.1 \pm 1.88$ \\
Size $(\mathrm{nm})$ & $131.7 \pm 11.8$ & $132.9 \pm 10.7$ & $133.5 \pm 13.6$ & $134.0 \pm 9.2$ & $137.1 \pm 12.3$ & $138.5 \pm 10.4$ & $140.3 \pm 13.2$ \\
\hline
\end{tabular}

Abbreviations: SD, standard deviation; $\mathrm{EE}$, entrapment efficiency.

Figure 3, the biodistribution of baicalin solution was observed in a time-dependent manner. From Figure 4, in the case of baicalin-loaded nanoliposomes, the biodistribution in plasma, heart, and lung was found to be in a time-dependent manner, whereas the biodistribution in liver, spleen, kidney, brain, and stomach was elevated within the first $2 \mathrm{~h}$ and afterward decreased at 2-24 $\mathrm{h}$ after intravenous administration. After intravenous administration of baicalin-loaded nanoliposomes, drug concentration in the lungs was the highest among the tested organs at all time points; moreover, drug concentration in the lungs was also significantly higher than that of its solution. For example, drug concentration of baicalin-loaded nanoliposomes showed 2.789-fold increase in the lungs at 15 min after intravenous administration in comparison with baicalin solutions. Whereas, drug concentration in the other organs was less than or similar to its solutions, suggesting a rapid and efficient localization of baicalin-loaded nanoliposomes in the lungs. In addition, extremely low plasma drug level and rapid plasma clearance were observed in the case of baicalin-loaded nanoliposomes after injection into rabbits in comparison with its solutions, which further supported the targeting characteristics of baicalin-loaded nanoliposomes to lungs.

To further understand the lung-targeting behavior of the drug when intravenously administered in the liposomal

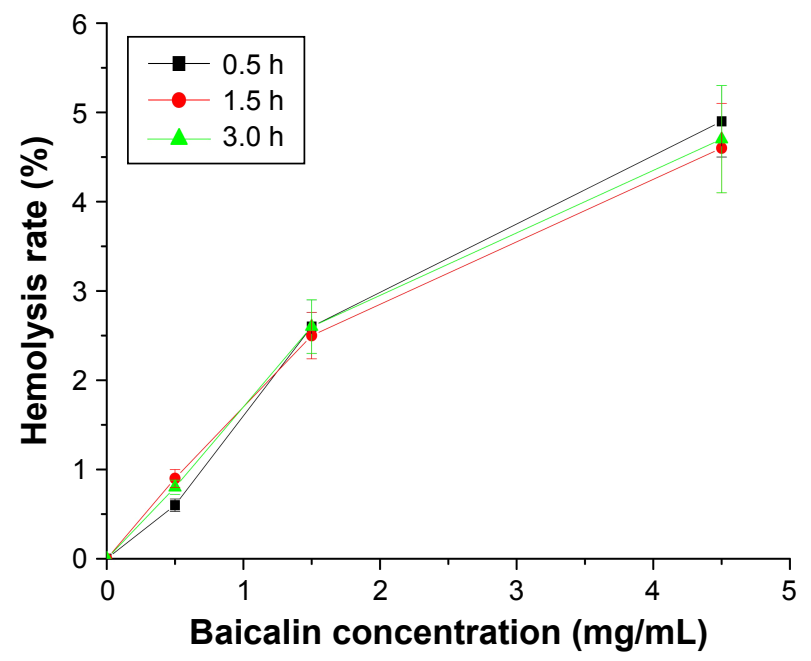

Figure 2 Hemolysis of erythrocytes by various drug concentrations of baicalinloaded nanoliposomes $(n=3)$. formulation, a comparative lung-targeting study was carried out between baicalin-loaded nanoliposomes and its solutions. On the basis of drug concentration in each tissue or plasma, the area under the curve (AUC) of the drug concentrationtime profile of baicalin and targeting parameters $\left(R_{e}, T_{e}\right.$, and $\mathrm{C}_{\mathrm{e}}$ ) was calculated and is shown in Table $3 .{ }^{22}$ Baicalin liposomal formulation showed the largest value of AUC for lung, which indicated that baicalin-loaded nanoliposomes developed in the present study were preferable for targeting to lungs than other organs.

As shown in Table 3, the value of $R_{e}$ (defined as $\mathrm{R}_{\mathrm{e}}=\mathrm{AUC}_{\text {liposomes }} / \mathrm{AUC}_{\text {solution }}$ ) for lungs was 4.837 in the case of baicalin-loaded nanoliposomes, which indicated that the exposure of the baicalin to lung was significantly increased by encapsulation in liposome carriers. We all know that when the value of $\mathrm{R}_{\mathrm{e}}$ is $>1$, the tissue is exposed to the drug to a greater extent when administered in the liposomal form. Thus, it suggested that the liposome carrier was more specific accumulation of the drug in the lungs in comparison to its solutions. The value of $\mathrm{T}_{\mathrm{e}}$ (defined as $\mathrm{R}_{\mathrm{e}}=\mathrm{AUC}_{\text {lung target }}$ $\mathrm{AUC}_{\text {other tissue }}$ ) demonstrated the efficiency of a given delivery system against the nontarget organ through the ratio of target to nontarget organ drug distribution. In this study, the $\mathrm{T}_{e}$ values of lung to other organs or plasma in the case of baicalin liposomal formulation all exceeded 1 and the value was 65.045 for plasma. Moreover, the ratios of $\mathrm{T}_{\mathrm{e} \text { liposomes }}$ $\mathrm{T}_{\text {e solution }}$ were increased significantly and the value was 14.131 for plasma. Therefore, the liposome carrier significantly increased the lung-targeting effect of baicalin in comparison with its solution. The value of $\mathrm{C}_{\mathrm{e}}$, defined as $\mathrm{C}_{\mathrm{e}}=\left(\mathrm{C}_{\max }\right)_{\text {liposomes }}$ ' $\left(\mathrm{C}_{\max }\right)_{\text {solution}}$, provided information regarding the efficiency of a delivery system on the change of biodistribution. Compared with baicalin solution, the value of $\mathrm{C}_{\mathrm{e}}$ in the lungs was increased by a factor of 2.789 in the case of its liposomal formulation, which indicated that baicalin-loaded nanoliposomes had a preference to concentrate drug in the lungs in comparison with its solutions.

In conclusion, these results of tissue distribution and lung-targeting evaluation demonstrated that baicalin-loaded nanoliposomes developed in this study had obvious lungtargeting characteristics as compared with its solutions. It is well known that the lungs, due to their susceptibility to some 


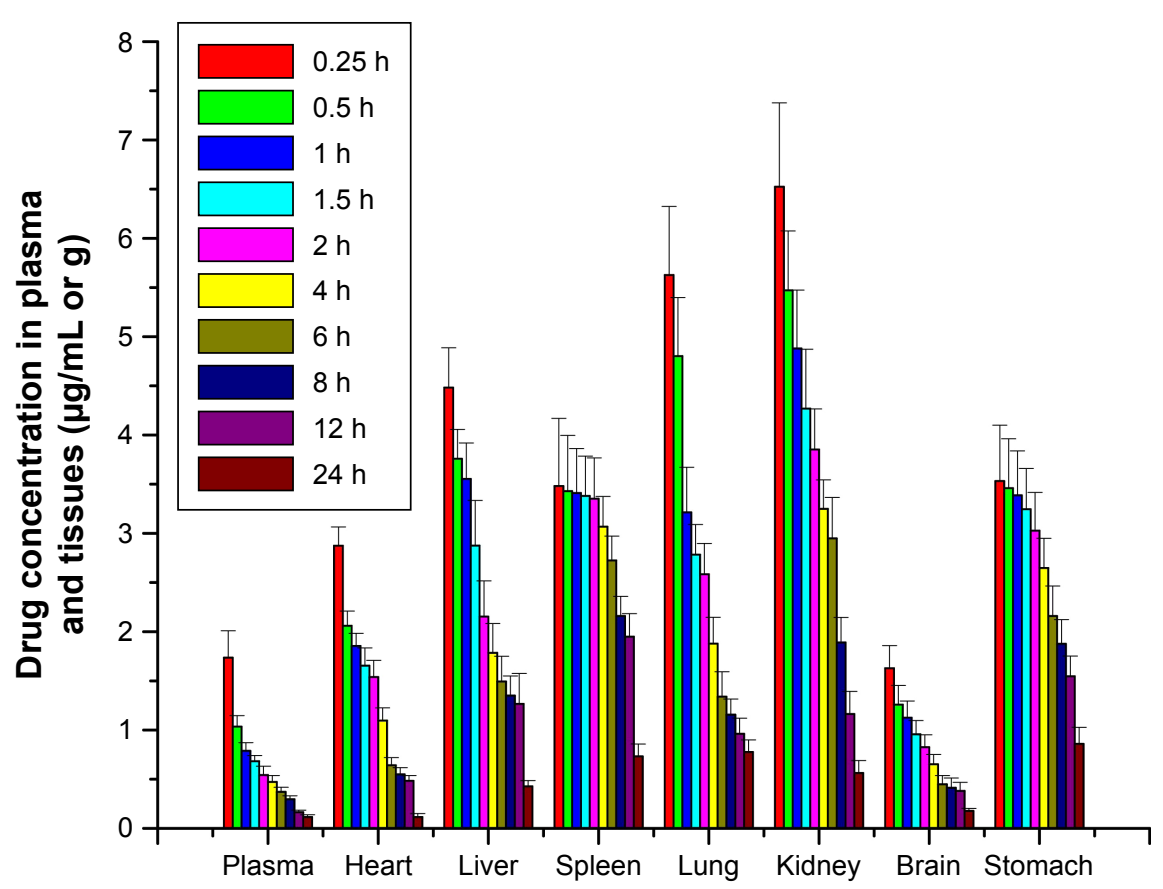

Figure 3 Distribution in rabbit tissues after intravenous administration of baicalin solution $(\bar{x} \pm S D, n=5$; plasma: $\mu g / m L$, tissues: $\mu g / g)$. Abbreviation: SD, standard deviation.

diseases such as lung cancer, tuberculosis, and pneumonia, are a suitable target organ to localize drugs. ${ }^{5}$ Therefore, the liposome carrier offered a possibility for baicalin for the treatment of lung cancer. Many studies showed that the liposomes accumulate mostly in the organs of the reticuloendothelial system such as liver, spleen, and lung within the first
15-30 min after intravenous administration of the liposomal formulation. ${ }^{5,23}$ In general, the liposomes with a particle size of $>5 \mu \mathrm{m}$ could be trapped passively by the vascular network of the lung to achieve lung-targeting effect. ${ }^{24}$ Besides the physiochemical characterization of liposomes such as particle size, surface charge, and membrane lipid component, 25,26

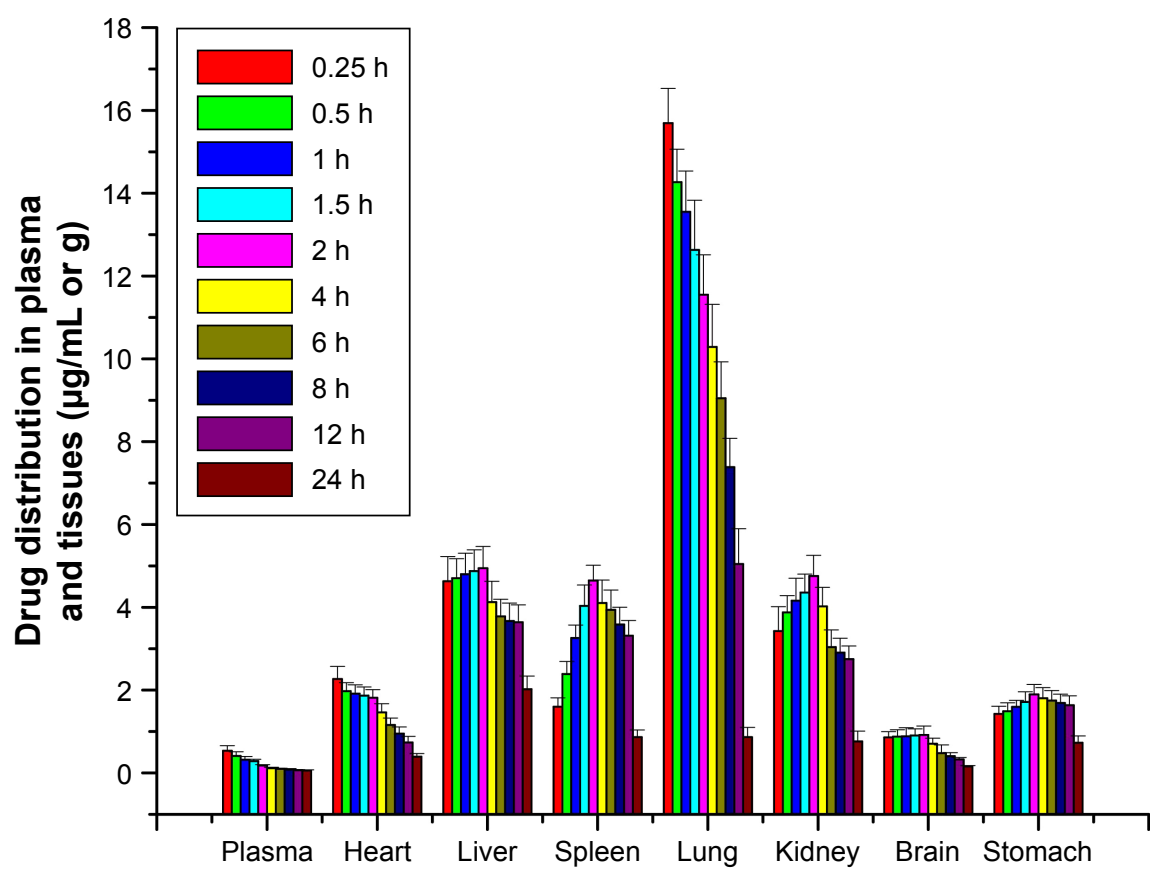

Figure 4 Distribution in rabbit tissues after intravenous administration of baicalin liposomes $(\bar{x} \pm S D, n=5$; plasma: $\mu g / m L$, tissues: $\mu g / g)$. Abbreviation: SD, standard deviation. 
Table 3 The lung-targeting parameters of baicalin liposomal formulation and its solution after intravenous administration in rabbit ${ }^{\mathrm{a}}$

\begin{tabular}{|c|c|c|c|c|c|c|c|}
\hline \multirow[t]{2}{*}{ Biosample } & \multicolumn{2}{|c|}{ AUC $(\mu \mathrm{g} \cdot \mathrm{h} / \mathrm{mL} \text { or } \mu \mathrm{g} \cdot \mathrm{h} / \mathrm{g})^{\mathrm{b}}$} & \multirow[t]{2}{*}{$\mathbf{R}_{\mathbf{e}}$} & \multicolumn{2}{|l|}{$T_{e}$} & \multirow[t]{2}{*}{ Ratio of $T_{e}$} & \multirow[t]{2}{*}{$\mathrm{C}_{\mathrm{e}}$} \\
\hline & Liposomes & Solution & & Liposomes & Solution & & \\
\hline Plasma & 2.42 & 7.07 & 0.342 & 65.045 & 4.603 & $14.13 \mid$ & 0.308 \\
\hline Heart & 22.10 & 15.23 & $1.45 \mathrm{I}$ & 7.123 & 2.137 & 3.333 & 0.789 \\
\hline Liver & 82.57 & 32.31 & 2.556 & 1.906 & 1.007 & 1.893 & 1.103 \\
\hline Spleen & 69.47 & 48.22 & 1.441 & 2.266 & 0.675 & 3.357 & 1.335 \\
\hline Lung & $|57.4|$ & 32.54 & 4.837 & 1.000 & 1.000 & 1.000 & 2.789 \\
\hline Kidney & 62.31 & 44.68 & 1.395 & 2.526 & 0.728 & 3.470 & 0.729 \\
\hline Stomach & 34.75 & 42.50 & 0.818 & 4.530 & 0.766 & 5.914 & 0.537 \\
\hline Brain & 9.84 & 10.72 & 0.918 & I5.997 & 3.035 & $5.27 \mid$ & 0.564 \\
\hline
\end{tabular}

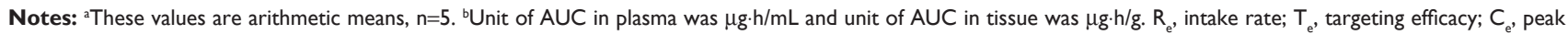
concentration ratio.

Abbreviation: AUC, area under the curve.

other factors such as particle-cell contact may also influence the biodistribution behavior of the drug. ${ }^{27}$ In this study, the highest distribution of baicalin with its nanoliposomes was observed in lungs. Specific lung-targeting mechanism needs further study.

\section{Evaluation of lung injury}

In the view of tissue distribution of baicalin, drug concentration and AUC in the lungs were significantly increased after intravenous administration of baicalin liposomal formulation to rabbits in comparison with its solution. In order to confirm the feasibility of baicalin-loaded nanoliposomes for lung-targeting delivery system, the possible lung injury induced by its liposomes was evaluated. The results showed that rats treated with baicalin-loaded nanoliposomes at the first, fourth, and seventh days after injection presented normal lung histology with normal sizes of airspaces and alveolar septa (Figure 5). Compared with the negative control group, no significant difference in lung changes at the first, fourth, and seventh days after injection of baicalin-loaded nanoliposomes to rats was observed. However, a slight broadening of alveolar septa was observed. Hence, the nanoliposomes seemed to induce some minor inflammation that did not increase with the time as observed from Figure $5 \mathrm{~A}-\mathrm{C}$.
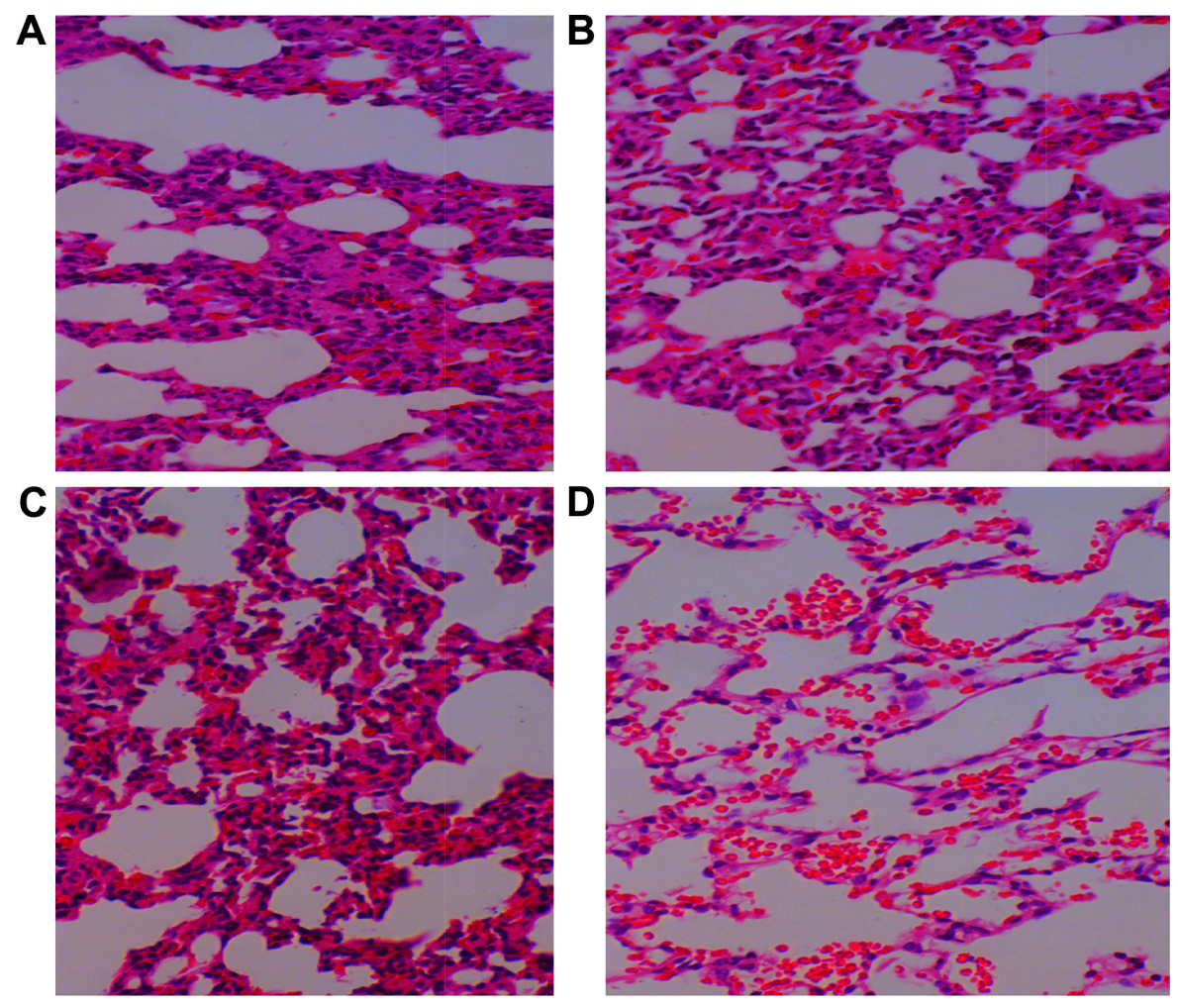

Figure 5 The pathological sections of lung at I (A), 4 (B), and 7 days (C) after intravenous administration of baicalin-loaded nanoliposomes and saline (D) as the control. Magnification $\times 200$. 


\section{Pharmacodynamics}

In order to evaluate the therapeutical efficacy of baicalinloaded nanoliposomes as a lung-targeting drug delivery system on lung cancer, the nude mice bearing orthotopic human lung cancer were intravenously administered with blank liposomes, baicalin solution, and baicalin-loaded nanoliposomes on every other day after 7 days post-tumor implantation. The results are shown in Figure 6. The total tumor weight of blank liposomes group from 11 to 12 days, baicalin solution group from 15 to 20 days and baicalin-loaded

A

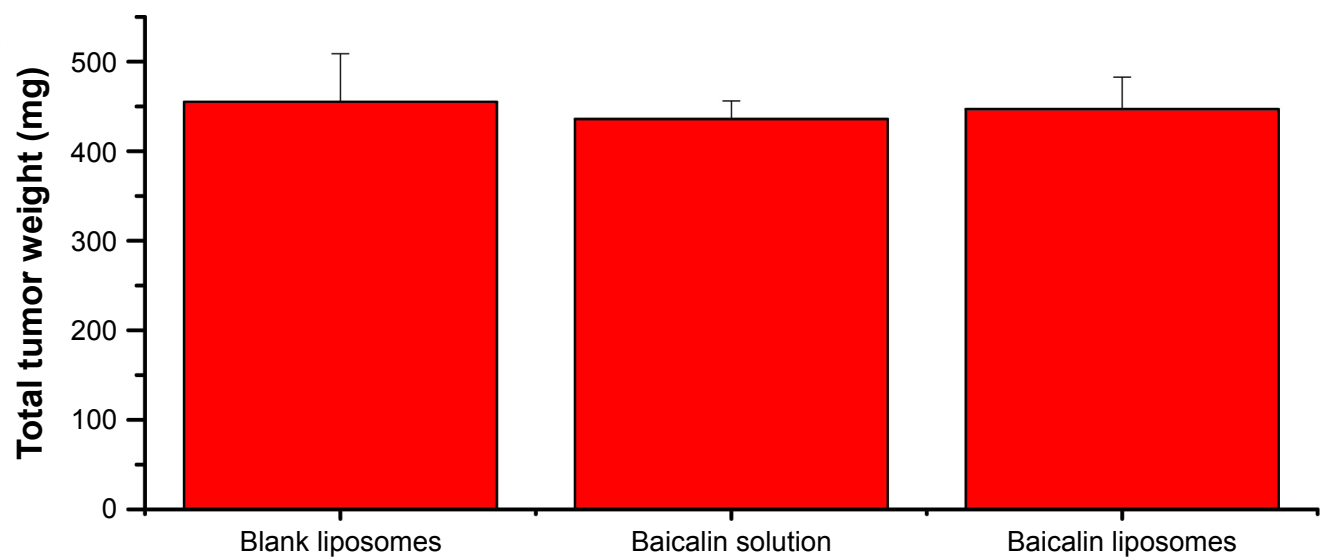

B
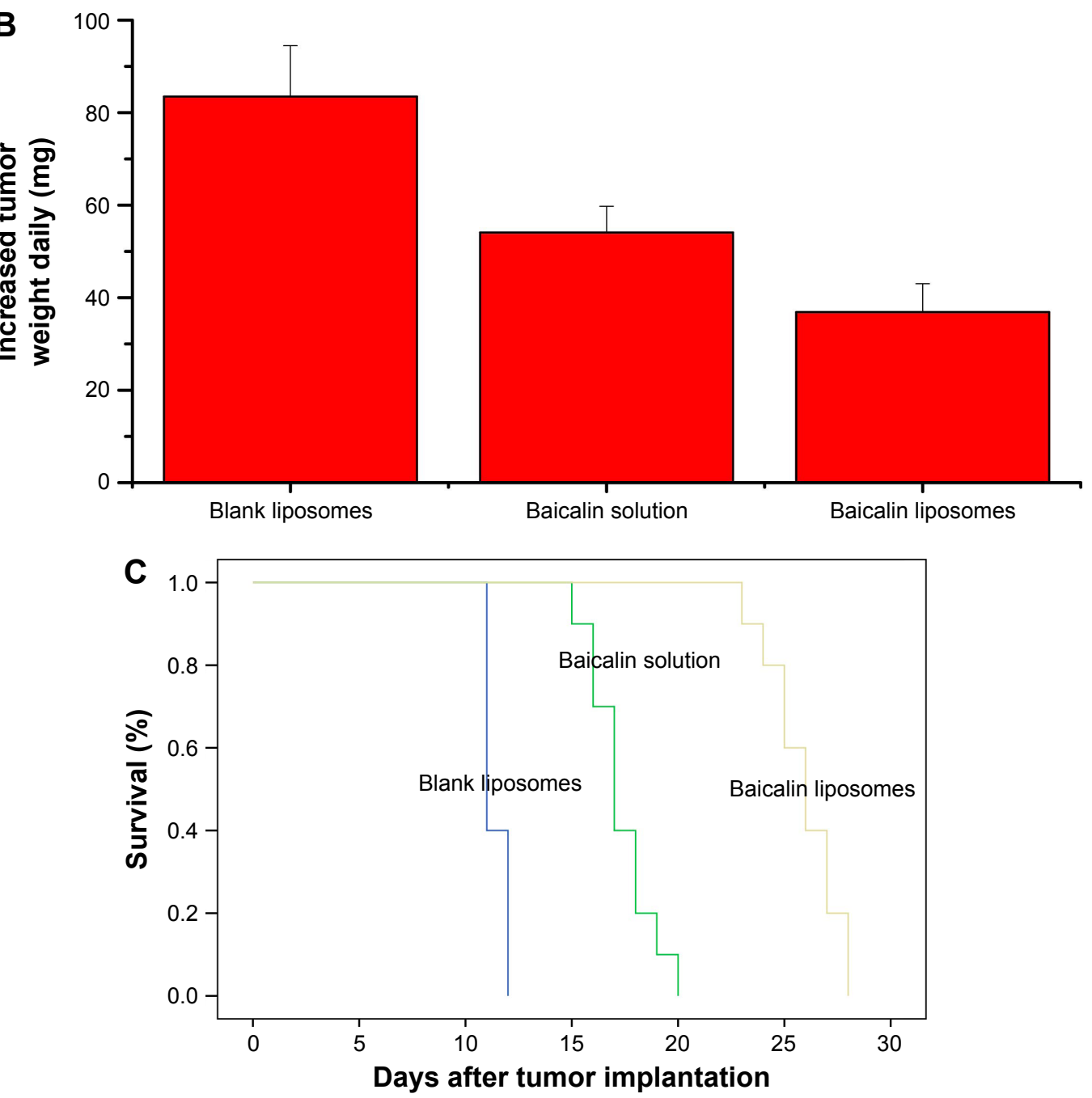

Figure 6 Pharmacodynamics in nude mice bearing orthotopic human lung cancer intravenously administered with baicalin-loaded nanoliposomes. Notes: (A) Total tumor weight of each group when the mice was naturally scarified. (B) Increased tumor weight daily of each group. (C) Survival rate study. 
nanoliposomes sample from 23 to 28 days after tumor implantation was $955.00 \pm 153.83 \mathrm{mg}, 936.00 \pm 120.18 \mathrm{mg}$, and $947.00 \pm 105.83 \mathrm{mg}$, respectively, which was not significantly different $(P>0.05$; Figure 6A). However, a significant difference in increased tumor weight daily of the saline group, baicalin solution group and baicalin-loaded nanoliposomes sample was observed. A significant decrease in increased tumor weight daily of baicalin-loaded nanoliposomes sample (36.89 $\pm 6.13 \mathrm{mg}$ tumor weight) was observed compared with blank liposomes (83.49 $\pm 11.01 \mathrm{mg}$ tumor weight) and baicalin solution groups (54.10 $\pm 5.64 \mathrm{mg}$ tumor weight; $P<0.01$; Figure 6B). Importantly, the nude mice bearing orthotopic human lung cancer treated with baicalin-loaded nanoliposomes presented demonstrated significant increased survival rates in comparison with blank liposomes and baicalin solution $(P<0.01$; Figure $6 \mathrm{C})$. The median survival time of tumor-bearing mice treated with blank liposomes, baicalin solution, and baicalin-loaded nanoliposomes was $11.40 \pm 0.16$ days, $17.30 \pm 0.47$ days, and $25.90 \pm 0.53$ days, respectively. The results were explained that baicalin-loaded nanoliposomes could deliver main drug into lung cancer based on the biodistribution study and greatly increased drug concentration in lung cancer. Therefore, baicalin-loaded nanoliposomes showed better efficacy in lung cancer treatment in comparison with baicalin solution with whole-body biodistribution.

\section{Conclusion}

Baicalin-loaded nanoliposomes for lung-targeting delivery system were successfully developed in this study for the first time. For in vitro evaluation, baicalin-loaded nanoliposomes exhibited high encapsulation efficiency, sustainedrelease behavior, and excellent stability. At the same time, the liposomes did not cause hemolytic activity. For in vivo evaluation, baicalin-loaded nanoliposomes can increase significantly drug concentration in the lungs after single intravenous administration. Moreover, according to the evaluation of lung injury, baicalin-loaded nanoliposomes were safe. Importantly, baicalin-loaded nanoliposomes showed better antitumor therapeutic efficacy in the nude mice bearing orthotopic human lung cancer. Therefore, the liposome is a promising drug carrier with excellent lung-targeting effect for Chinese medicine monomer, like baicalin, for instance.

\section{Acknowledgment}

This study was financially supported by the National Natural Science Foundation of China (81101678, 81341124, 31201566), the Science and Technology Support Project of Sichuan Province (2014SZ0071, 2014FZ0105), the Joint Fund of Sichuan Province, Luzhou City and Southwest Medical University (14JC0134, 14ZC0026, 14ZC006-6) and the Joint Fund of Luzhou City and Southwest Medical University (2013LZLY-K80, 2015LZCYD-S09 (4/8)).

\section{Disclosure}

The authors report no conflicts of interest in this work.

\section{References}

1. Zhuang B, Du L, Xu H, et al. Self-assembled micelle loading cabazitaxel for therapy of lung cancer. Int J Pharm. 2016;499(1-2):146-155.

2. Melguizo C, Cabeza L, Prados J, et al. Enhanced antitumoral activity of doxorubicin against lung cancer cells using biodegradable poly(butylcyanoacrylate) nanoparticles. Drug Des Devel Ther. 2015;9: 6433-6444.

3. Li D, Gong L. Preparation of novel pirfenidone microspheres for lungtargeted delivery: in vitro and in vivo study. Drug Des Devel Ther. 2016;10:2815-2821.

4. Mehta HJ, Patel V, Sadikot RT. Curcumin and lung cancer - a review. Target Oncol. 2014;9(4):295-310.

5. Wei Y, Zhao L. Passive lung-targeted drug delivery systems via intravenous administration. Pharm Dev Technol. 2014;19(2):129-136.

6. Zhao L, Wei Y, Huang Y, He B, Zhou Y, Fu J. Nanoemulsion improves the oral bioavailability of baicalin in rats: in vitro and in vivo evaluation. Int J Nanomedicine. 2013;8:3769-3779.

7. Wei Y, Pi C, Yang G, et al. LC-UV determination of baicalin in rabbit plasma and tissues for application in pharmacokinetics and tissue distribution studies of baicalin after intravenous administration of liposomal and injectable formulations. Molecules. 2016;21(4):E444.

8. Hu YZ, Wang DH, Luan Y, Gong HD. Antitumor effect of baicalin on rat brain glioma. Zhonghua Zhong Liu Za Zhi. 2013;35(1):11-16.

9. Wang CZ, Zhang CF, Chen L, Anderson S, Lu F, Yuan CS. Colon cancer chemopreventive effects of baicalein, an active enteric microbiome metabolite from baicalin. Int J Oncol. 2015;47(5):1749-1758.

10. Yu Y, Pei M, Li L. Baicalin induces apoptosis in hepatic cancer cells in vitro and suppresses tumor growth in vivo. Int J Clin Exp Med. 2015; 8(6):8958-8967.

11. Du G, Han G, Zhang S, et al. Baicalin suppresses lung carcinoma and lung metastasis by SOD mimic and HIF-1alpha inhibition. Eur J Pharmacol. 2010;630(1-3):121-130.

12. Meng H, Xu Y. Pirfenidone-loaded liposomes for lung targeting: preparation and in vitro/in vivo evaluation. Drug Des Devel Ther. 2015;9: 3369-3376.

13. Ji C, Na W, Fei X, Sheng-Jun C, Jia-Bi Z. Characterization, lung targeting profile and therapeutic efficiency of dipyridamole liposomes. J Drug Target. 2006;14(10):717-724.

14. Harsha S, Al-Khars M, Al-Hassan M, et al. Pharmacokinetics and tissue distribution of spray-dried carboplatin microspheres: lung targeting via intravenous route. Arch Pharm Res. 2014;37(3):352-360.

15. Ramaiah B, Nagaraja SH, Kapanigowda UG, Boggarapu PR, Subramanian R. High azithromycin concentration in lungs by way of bovine serum albumin microspheres as targeted drug delivery: lung targeting efficiency in albino mice. Daru. 2016;24(1):14.

16. Lee SY, Jung E, Park JH, et al. Transient aggregation of chitosanmodified poly(d,l-lactic-co-glycolic) acid nanoparticles in the blood stream and improved lung targeting efficiency. J Colloid Interface Sci. 2016;480:102-108.

17. Anselmo AC, Gupta V, Zern BJ, et al. Delivering nanoparticles to lungs while avoiding liver and spleen through adsorption on red blood cells. ACS Nano. 2013;7(12):11129-11137.

18. Hong Y, He W, Li D, He L, Zhang W. Preparation and in vitro anti-tumor effect of baicalin liposome. Chin J Exp Tradit Med Formulae. 2012; 18(3):29-31. 
19. Chen YJ, Jia Y, Jin R, et al. Preparation of baicalin flexible nanoliposomes. Chin J Exp Tradit Med Formulae. 2011;17(5):1-4.

20. Liu S, Wu WY, Xi ZX, Wang N, Huang J. Determination of in vitro drug release of baicalin liposomes by HPLC. Chin Pharm. 2007;18(33): 2594-2596.

21. Mu YM. Preparation and characterization of liposomes containing baicalin. Chin Tradit Patent Med. 2008;30(7):1077-1079.

22. Gupta PK, Hung CT. Quantitative evaluation of targeted drug delivery systems. Int J Pharm. 1989;56(3):217-226.

23. Waser PG, Muller U, Kreuter J, et al. Localization of colloidal particles (liposomes, hexylcyanoacrylate nanoparticles and albumin nanoparticles) by histology and autoradiography in mice. Int J Pharm. 1987; 39(3):213-227.

24. Zhang SX, Gao XJ, Shen KH, Yang PW, Ju XL. Evaluation of poly (D,L-lactideco-glycolide) microspheres for the lung-targeting of yuanhuacine, a novel DNA topoisomerase I inhibitor. J Drug Target. 2009;17(4):286-293.
25. Hirano K, Hunt CA. Lymphatic transport of liposome-encapsulated agents: effects of liposome size following intraperitoneal administration. J Pharm Sci. 1985;74(9):915-921.

26. Kim CK, Lee MK, Han JH, Lee BJ. Pharmacokinetics and tissue distribution of methotrexate after intravenous injection of differently charged liposome-entrapped methotrexate to rats. Int J Pharm. 1994; 108(1):21-29.

27. Xiang QY, Wang MT, Chen F, et al. Lung-targeting delivery of dexamethasone acetate loaded solid lipid nanoparticles. Arch Pharm Res. 2007;30(4):519-525.
International Journal of Nanomedicine

\section{Publish your work in this journal}

The International Journal of Nanomedicine is an international, peerreviewed journal focusing on the application of nanotechnology in diagnostics, therapeutics, and drug delivery systems throughout the biomedical field. This journal is indexed on PubMed Central,

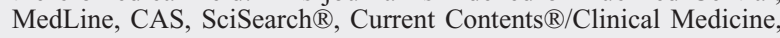

\section{Dovepress}

Journal Citation Reports/Science Edition, EMBase, Scopus and the Elsevier Bibliographic databases. The manuscript management system is completely online and includes a very quick and fair peer-review system, which is all easy to use. Visit http://www.dovepress.com/ testimonials.php to read real quotes from published authors.

Submit your manuscript here: http://www.dovepress.com/international-journal-of-nanomedicine-journal 